\title{
A permanganate mediated approach to the synthesis of cis-solamin
}

\author{
Alexander R. L. Cecil and Richard C. D. Brown \\ Department of Chemistry, University of Southampton, Highfield, Southampton, SO17 1BJ, UK \\ E-mail: rcb1@soton.ac.uk
}

(received 12 Nov 01; accepted 10 Mar 02; published on the web 18 Mar 02)

\begin{abstract}
A model study directed towards the synthesis of the Annonaceous acetogenin cis-solamin (1) is reported. The key step in the synthesis involved the permanganate-promoted oxidative cyclisation of ethyl (E)-7-methylocta-2,6-dienoate (7) to afford a tetrahydrofuran diol $\mathbf{6}$. The remaining $\mathrm{C}_{1}-\mathrm{C}_{13}$ portion of the target was introduced using an organocopper-mediated opening of epoxide 5, followed by a ruthenium-catalysed Alder-ene reaction. Significantly, no protecting groups were required during the assembly of the fragments.
\end{abstract}

Keywords: Permanganate, cis-solamin, oxidative cyclization, furan, epoxide, ene reaction

\section{Introduction}

The Annonaceous acetogenins are natural products isolated from the plant family, Annonaceae (custard-apple family). They are derived from C32 or C34 fatty acids combined with a 2propanol unit at $\mathrm{C} 2$ that typically forms part of a terminal $\alpha, \beta$-unsaturated $\gamma$-lactone (butenolide). In addition, one or more 2,5-disubstituted tetrahydrofuran (THF) rings are often present in the hydrocarbon backbone, which may carry oxygen substituents or unsaturation along its length. Considerable interest has been shown in the Annonaceous acetogenins, largely due to their cytotoxicity both towards healthy cells, but particularly cancerous cells. ${ }^{1,2}$ Their mechanism of action involves ATP deprivation as a result of inhibition of the NADH: ubiquinone oxoreductase present in complex I of the mitochondrial electron transport system, and the ubiquinone-linked NADH oxidase that is active in plasma membranes of cancerous cells. ${ }^{3}$ Other biological properties such as immunosuppressive, pesticidal, antiprotozoal, antifeedant, anthelmintic and antimicrobial activities have been reported, ${ }^{1,4-6}$ further fuelling interest in these natural products. A substantial number of total syntheses of Annonaceous acetogenins have now been published, ${ }^{1,7}$ and some studies towards the synthesis of biologically active analogues have appeared. ${ }^{8-10}$ Most of the synthetic work to date has focused on the preparation of compounds with trans-2,5disubstituted THF rings, whereas the synthesis of the cis-isomers has been comparatively neglected. ${ }^{11,12}$ 
Here we describe an approach to the synthesis of the core and right hand portions of the Annonaceous acetogenin cis-solamin (1). Our strategy centres on the use of a permanganate oxidative cyclisation of a 1,5-diene to produce the cis-2,5-disubstituted THF diol core (Scheme 1). ${ }^{13-16}$ In this way, four of the five stereocentres present within the target molecule will be introduced with control of relative stereochemistry in a single synthetic step. Ultimately, control of absolute stereochemistry will be achieved using a chiral auxiliary. ${ }^{17-18}$

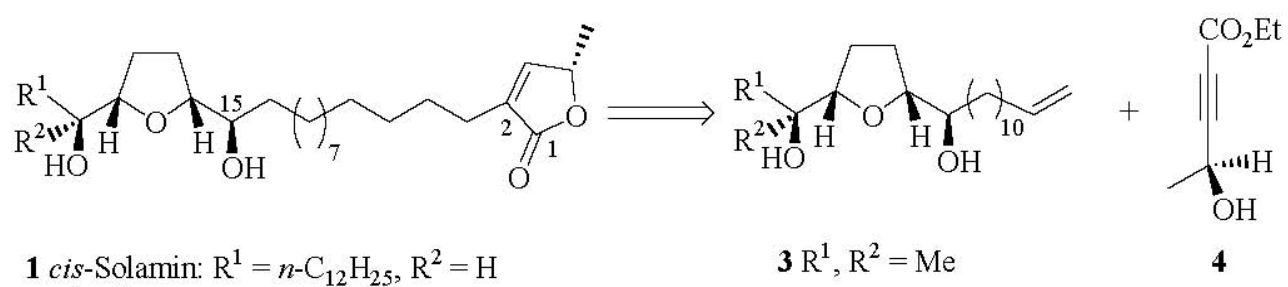

$2 \mathrm{R}^{1}, \mathrm{R}^{2}=\mathrm{Me}$

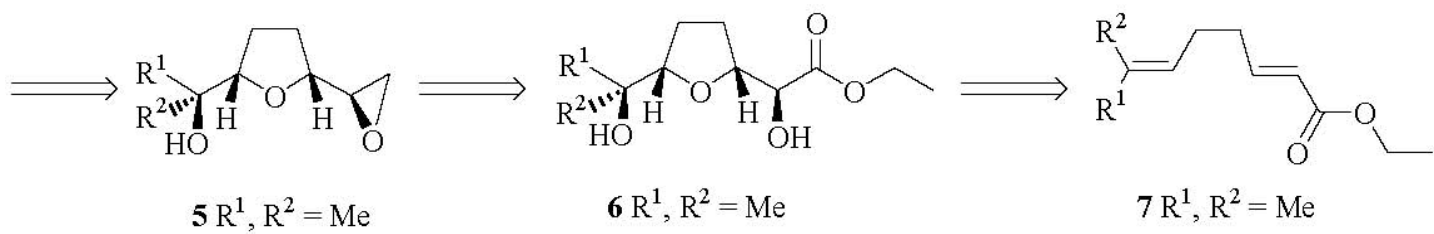

Scheme 1. Approach to cis-2,5-disubstituted THF-containing acetogenins employing a permanganate-promoted oxidative cyclisation.

The butenolide ring present in 2 would be prepared using a ruthenium-catalysed Alder-ene reaction developed by Trost. ${ }^{19}$ Introduction of the required alkenyl chain would be carried out using an organocopper mediated opening of epoxide 5, which in turn would derive from ester 6.

\section{Results and Discussion}

Our synthesis of the model compound 2 began with the 1,5-diene 7, prepared in two steps from 2-methylbut-3-en-2-ol. ${ }^{20,21}$ Oxidative cyclisation of diene 7 gave the desired THF diol $\mathbf{6}$ in good yield $(63 \%)$ as a single diastereoisomer. ${ }^{22}$ Reduction of ester 6 produced the polar triol $\mathbf{8}$, which underwent selective tosylation to afford diol $\mathbf{9}$. Formation of the key epoxide intermediate $\mathbf{5}$ occurred rapidly when a methylene chloride solution of 9 was treated with DBU. The C3-C13 carbon atoms present in the target were introduced using a copper-catalysed Grignard reaction to give the terminal alkene 3 , required for the formation of the butenolide ring in the subsequent step.

The Trost ruthenium-catalysed Alder-ene reaction between alkene 3 and optically enriched alkyne $4^{\ddagger}$ gave the desired diastereoisomeric butenolides 10A and 10B (indistinguishable by $\mathrm{nmr}$ ) in satisfactory yield in a 5:1 ratio with the uncyclised regioisomer which is typically observed in these reactions. ${ }^{19}$ It is noteworthy that the use of protecting groups was avoided 
during the assembly of the fragments, with both the copper-catalysed Grignard opening of epoxide 5 and the ruthenium-catalysed Alder-ene reaction proceeding in the presence of free hydroxyl groups.
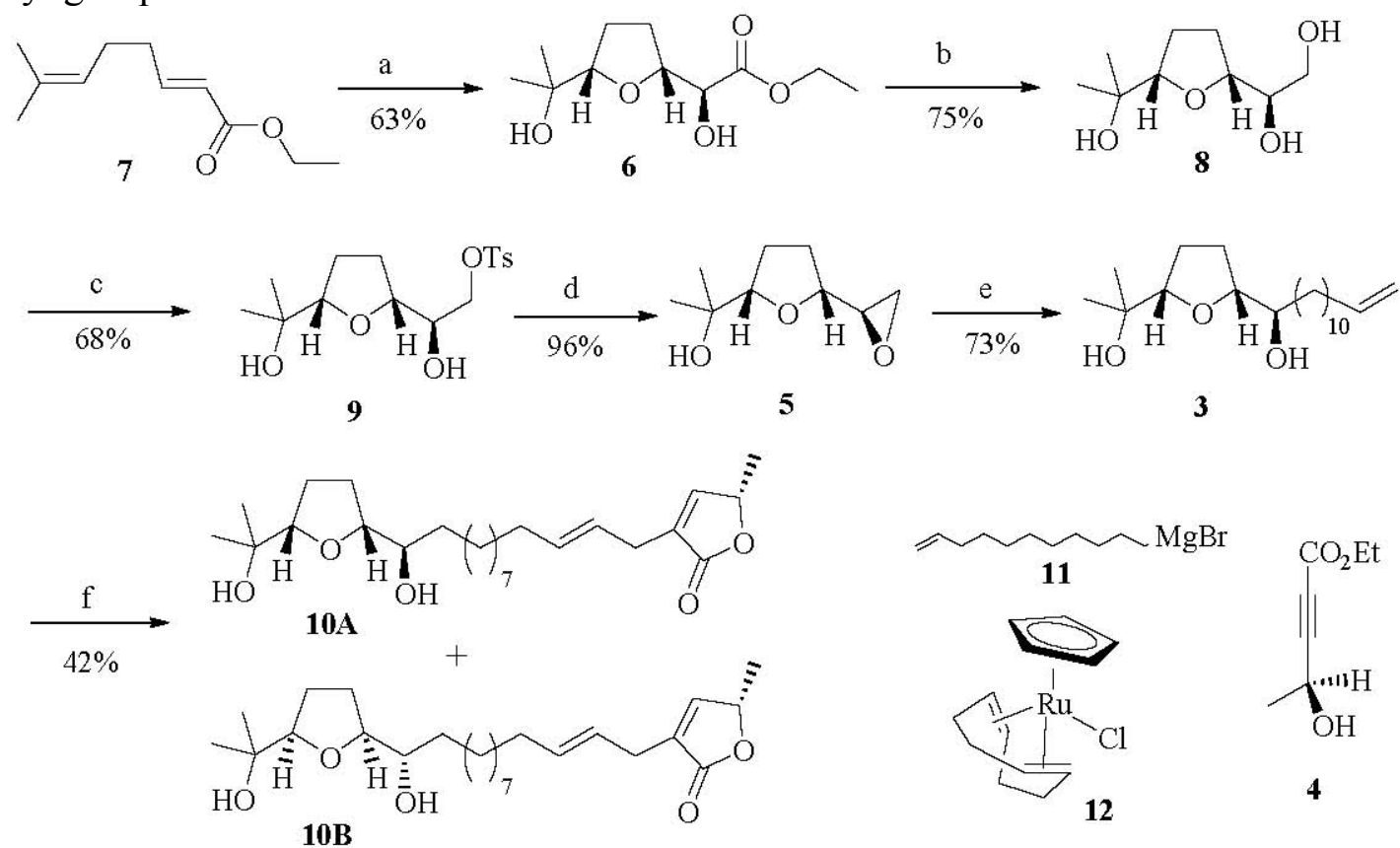

Scheme 2. Synthesis of model Annonaceous acetogenin 2.

Reagents and conditions: a) $\mathrm{KMnO}_{4}, \mathrm{AcOH}$, buffer $\mathrm{pH} 6.5$, acetone, $-20{ }^{\circ} \mathrm{C}$; b) $\mathrm{NaBH}_{4}, \mathrm{THF}$, $\mathrm{H}_{2} \mathrm{O}$; c) TsCl, DMAP, $\mathrm{Et}_{3} \mathrm{~N}, \mathrm{CH}_{2} \mathrm{Cl}_{2}$; d) $\mathrm{DBU}, \mathrm{CH}_{2} \mathrm{Cl}_{2}$; e) 11, CuI, THF; f) 4, $\mathrm{CpRu}(\mathrm{COD}) \mathrm{Cl}$ (12), benzene/EtOH, sealed vessel, $\Delta$.

With compound $\mathbf{1 0}$ in hand, we were in a position to investigate the selective reduction of the disubstituted olefin. The reduction was carried out in a steel bomb at 2-4 atmospheres of $\mathrm{H}_{2}$, giving a 3:2 mixture (nmr) of the desired butenolide 2 (two diastereoisomers) and over reduced lactone 13 (possible 4 diastereoisomers) in near quantitative yield. Although efforts to separate 2 and 13 were unsuccessful, we are confident that the over reduction observed here will be avoided when we come to tackle cis-solamin itself by more careful control of $\mathrm{H}_{2}$ pressure. $^{23}$ Unfortunately, we were not able to demonstrate this on the model substrate, as insufficient material was available to attempt the reaction a second time.

In conclusion, a short synthesis to the central THF ring and right hand side-chain of cissolamin has been achieved. In the key step a permanganate oxidative cyclisation of a 1,5-diene was used to generate four of the five stereocentres present in the natural product. The THF diol functionality was shown to be compatible with the organocopper and ruthenium catalysed Alder-ene reactions that will ultimately be used in the synthesis of cis-solamin. An asymmetric synthesis of cis-solamin by this approach is currently under investigation. 


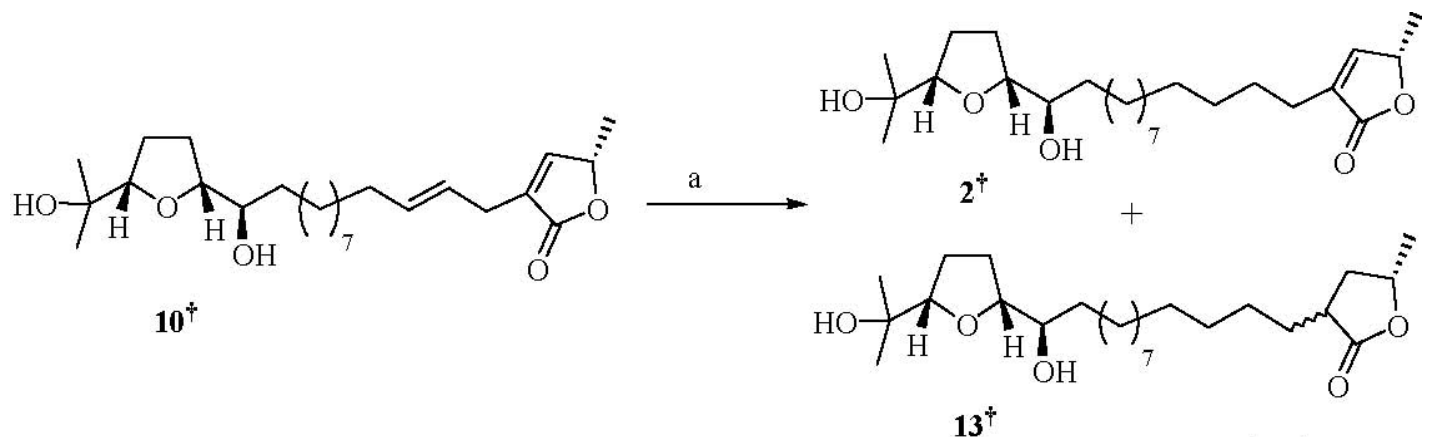

Scheme 3 Attempted selective reduction of $\mathbf{1 0}$.

Ratio $\mathbf{2} / \mathbf{1 3}=\mathbf{3}: 2$

Reagents and conditions: a) $\mathrm{Rh}\left(\mathrm{PPh}_{3}\right)_{3} \mathrm{Cl}, \mathrm{H}_{2}$ (2-4 bar), benzene/EtOH.

\section{Experimental Section}

General Methods. ${ }^{1} \mathrm{H}-\mathrm{NMR}$ and ${ }^{13} \mathrm{C}-\mathrm{NMR}$ were recorded on 300 or $400 \mathrm{MHz}$ spectrometers (300 or $400 \mathrm{MHz},{ }^{1} \mathrm{H}-\mathrm{NMR}$ respectively and 75 or $100 \mathrm{MHz},{ }^{13} \mathrm{C}-\mathrm{NMR}$ respectively) in deuterated chloroform $\left(\mathrm{CDCl}_{3}\right)$ with chloroform $\left(\delta 7.26 \mathrm{ppm}{ }^{1} \mathrm{H}, \delta 77.5 \mathrm{ppm}{ }^{13} \mathrm{C}\right)$ as the internal standard unless stated otherwise. IR spectra were recorded on a Nicolet Impact 400 spectrometer, fitted with a Spectra-Tech Thunderdome accessory. The abbreviations s (strong), $\mathrm{m}$ (medium), w (weak) and br (broad) are used when reporting the data. Melting points were obtained in open capillary tubes and are uncorrected. All non-aqueous reactions were carried out under an inert atmosphere of $\mathrm{N}_{2}$, in oven-dried glassware unless otherwise stated. The following solvents were distilled before use: THF (from Na/benzophenone) and $\mathrm{CH}_{2} \mathrm{Cl}_{2}$ (from $\mathrm{CaH}_{2}$ ) and where appropriate, other reagents and solvents were purified by standard techniques. ${ }^{24} \mathrm{TLC}$ was performed on aluminium-backed plates coated with silica gel 60 with an $\mathrm{F}_{254}$ indicator; the chromatograms were visualised under UV light and/or by staining with phosphomolybdic acid (20\% solution in ethanol) or $\mathrm{KMnO}_{4}$. Flash column chromatography was performed with 40-63 $\mu \mathrm{m}$ silica gel (Merck). The buffer solution used in the permanganate oxidative cyclisation is an aqueous $4: 1$ mixture of $1 / 16 \mathrm{M} \mathrm{KH}_{2} \mathrm{PO}_{4} \& 1 / 16 \mathrm{M} \mathrm{Na}_{2} \mathrm{HPO}_{4}$ at $\mathrm{pH} 6.5$.

Ethyl (2S*)-2-hydroxy-2-[(2R*,5S*)-5-(1-hydroxy-1-methylethyl)tetrahydro-2-furanyl]ethanoate (6). A solution of $0.4 \mathrm{M} \mathrm{KMnO}_{4}(6.60 \mathrm{~mL}, 2.6 \mathrm{mmol}, 1.6 \mathrm{eq})$ and acetic acid $(265 \mu \mathrm{L}, 4.6 \mathrm{mmol}$, $2.8 \mathrm{eq}$ ) was added dropwise over $10 \mathrm{~min}$ to a mixture of diene 7 (300 $\mathrm{mg}, 1.7 \mathrm{mmol}$ ), buffer $(1.16 \mathrm{~mL})$ and acetone $(20 \mathrm{~mL})$ at $-20{ }^{\circ} \mathrm{C}$ (internal). The reaction was quenched after a further 2 min by addition of ice-cooled $\mathrm{Na}_{2} \mathrm{~S}_{2} \mathrm{O}_{5}$ (sat. aq, $\left.40 \mathrm{~mL}\right)$ and ice $(20 \mathrm{~g})$. Ether $(50 \mathrm{~mL}), \mathrm{NaCl}(10$ g) and $\mathrm{NaHCO}_{3}(\mathrm{aq}, 20 \mathrm{~mL}$ ) were added and the organic layer separated. The aqueous layer was extracted with $\mathrm{CH}_{2} \mathrm{Cl}_{2}(2 \times 30 \mathrm{~mL})$. The organic layers were combined and dried $\left(\mathrm{Na}_{2} \mathrm{SO}_{4}\right)$, before concentrating in vacuo to give a yellow oil $(350 \mathrm{mg})$. Purification by column chromatography on silica gel (3:72:33:2 EtOAc/hexane) gave the title compound 6 (241 mg, 1.0 mmol, $63 \%$ ) as a white solid. Recrystallisation from EtOAc/hexane gave white crystals of mp 
44-45 ${ }^{\circ} \mathrm{C}$; ${ }^{1} \mathrm{H}-\mathrm{NMR}\left(300 \mathrm{MHz}, \mathrm{CDCl}_{3}\right) \delta 4.43(1 \mathrm{H}, \mathrm{ddd}, J=2.2,4.4,6.6 \mathrm{~Hz}, \mathrm{CHCHOH}), 4.34-$ $4.21\left(2 \mathrm{H}, \mathrm{m}, \mathrm{OCH}_{2} \mathrm{CH}_{3}\right), 4.14\left(1 \mathrm{H}, \mathrm{d}, J=2.0 \mathrm{~Hz}, \mathrm{CHCO}_{2}\right), 3.78(1 \mathrm{H}, \mathrm{t}, J=7.0 \mathrm{~Hz}$, $\left.\mathrm{CHC}\left(\mathrm{CH}_{3}\right)_{2}\right), 3.22(2 \mathrm{H}, \mathrm{br}, \mathrm{OH}), 2.20-1.83$ (4H, m, $\left.\mathrm{CH}_{2} \mathrm{CH}_{2}(\mathrm{THF})\right), 1.34$ (3H, t, J = $7.2 \mathrm{~Hz}$, $\left.\mathrm{CH}_{3} \mathrm{CH}_{2} \mathrm{O}\right) 1.29\left(3 \mathrm{H}, \mathrm{s},\left(\mathrm{CH}_{3}\right)_{2} \mathrm{COH}\right), 1.15\left(3 \mathrm{H}, \mathrm{s},\left(\mathrm{CH}_{3}\right)_{2} \mathrm{COH}\right) ;{ }^{13} \mathrm{C}-\mathrm{NMR}\left(75 \mathrm{MHz}, \mathrm{CDCl}_{3}\right) \delta$ $173.6(\mathrm{C}), 86.7(\mathrm{CH}), 80.0(\mathrm{CH}), 74.0(\mathrm{CH}), 72.1(\mathrm{C}), 61.9\left(\mathrm{CH}_{2}\right), 28.2\left(\mathrm{CH}_{2}\right), 28.1\left(\mathrm{CH}_{3}\right), 26.3$ $\left(\mathrm{CH}_{2}\right), 25.2\left(\mathrm{CH}_{3}\right), 14.3\left(\mathrm{CH}_{3}\right)$; IR $v$ 3350(br), 1737(s), 1364(s), 1264(s), 1199(s), 1158(s), 1121(s), 1078(s), 1026(s) $\mathrm{cm}^{-1}$; LRMS (CI) m/z $233\left(10 \%,[\mathrm{M}+\mathrm{H}]^{+}\right), 250\left(20 \%,\left[\mathrm{M}+\mathrm{NH}_{4}\right]^{+}\right)$, $\left.215\left(100 \% \text {, [M+H-H } \mathrm{H}_{2} \mathrm{O}\right]^{+}\right)$Da; HRMS (EI) calcd for $\mathrm{C}_{11} \mathrm{H}_{21} \mathrm{O}_{5} \mathrm{~m} / \mathrm{z}$ 233.1389, found 233.1391.

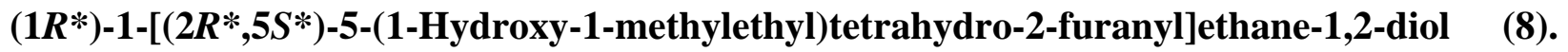
To a solution of ester $6(200 \mathrm{mg}, 0.86 \mathrm{mmol})$ and $\mathrm{NaBH}_{4}(66 \mathrm{mg}, 1.7 \mathrm{mmol})$ in THF (25 mL) at rt was added $\mathrm{H}_{2} \mathrm{O}(0.5 \mathrm{~mL})$. After $16 \mathrm{~h}$ a $1: 1$ solution of $\mathrm{MeOH} / \mathrm{CH}_{2} \mathrm{Cl}_{2}(8 \mathrm{~mL})$ was added. After $10 \mathrm{~min}$ the solution was concentrated in vacuo to give an oil. Purification by column chromatography on silica gel $\left(1: 191: 9 \mathrm{MeOH} / \mathrm{CH}_{2} \mathrm{Cl}_{2}\right)$ gave the title triol 8 (123 mg, $0.65 \mathrm{mmol}$, $75 \%)$ as a white solid. Recrystallisation from EtOAc/hexane gave white crystals of mp 49-51 ${ }^{\circ} \mathrm{C}$; ${ }^{1} \mathrm{H}-\mathrm{NMR}\left(300 \mathrm{MHz}, \mathrm{CDCl}_{3}\right) \delta 4.11-4.05(1 \mathrm{H}, \mathrm{m}, \mathrm{CHCHOH}), 3.80(1 \mathrm{H}, \mathrm{t}, J=7.0 \mathrm{~Hz}$, $\left.\mathrm{CHC}\left(\mathrm{CH}_{3}\right)_{2} \mathrm{OH}\right), 3.74-3.60\left(2 \mathrm{H}, \mathrm{m}, \mathrm{CH}_{2} \mathrm{OH}\right), 3.56-3.49(1 \mathrm{H}, \mathrm{m}, \mathrm{CHOH}), 2.53(3 \mathrm{H}$, br s, OH), 2.10-1.83 (4H, m, $\mathrm{CH}_{2}$ (THF)), $1.20\left(3 \mathrm{H}, \mathrm{s},\left(\mathrm{CH}_{3}\right)_{2} \mathrm{COH}\right), 1.05\left(3 \mathrm{H}, \mathrm{s},\left(\mathrm{CH}_{3}\right)_{2} \mathrm{COH}\right) ;{ }^{13} \mathrm{C}-\mathrm{NMR} \delta$ $\left(75 \mathrm{MHz}, \mathrm{CDCl}_{3}\right) 86.7(\mathrm{CH}), 80.2(\mathrm{CH}), 74.2(\mathrm{CH}), 72.0(\mathrm{C}), 65.3\left(\mathrm{CH}_{2}\right), 28.5\left(\mathrm{CH}_{2}\right), 27.9$ $\left(\mathrm{CH}_{3}\right), 26.0\left(\mathrm{CH}_{2}\right), 25.4\left(\mathrm{CH}_{3}\right)$; IR $v$ 3442(br), 3309(br), 3139(br), 1163(s), 1152(s), 1129(s), 1080(s), 1066(s), 1050(s) cm ${ }^{-1}$; LRMS (CI) m/z $191\left(10 \%,[\mathrm{M}+\mathrm{H}]^{+}\right), 208\left(20 \%,\left[\mathrm{M}+\mathrm{NH}_{4}\right]^{+}\right)$, $173\left(100 \%,\left[\mathrm{MH}^{+}-\mathrm{H}_{2} \mathrm{O}\right]\right)$; HRMS (EI) calcd for $\mathrm{C}_{9} \mathrm{H}_{19} \mathrm{O}_{4} \mathrm{~m} / \mathrm{z}$ 191.1283, found 191.1280.

(2R*)-2-Hydroxy-2-[(2R*,5S*)-5-(1-hydroxy-1-methylethyl)tetrahydro-2-furanyl]ethyl 1benzenesulfonate (9). To a solution of triol 8 (55 mg, $0.29 \mathrm{mmol})$, tosyl chloride (110 $\mathrm{mg}, 0.58$ mmol) and DMAP (4 mg, $0.03 \mathrm{mmol})$ in $\mathrm{CH}_{2} \mathrm{Cl}_{2}(10 \mathrm{~mL})$ was added $\mathrm{Et}_{3} \mathrm{~N}(0.3 \mathrm{~mL}, 2 \mathrm{mmol})$. After $16 \mathrm{~h} \mathrm{CH}_{2} \mathrm{Cl}_{2}(40 \mathrm{~mL})$ and brine $(20 \mathrm{~mL})$ were added, the organic phase was separated, dried $\left(\mathrm{Na}_{2} \mathrm{SO}_{4}\right)$ and concentrated in vacuo to give a colourless oil (160 mg). Purification by column chromatography using silica gel (3:7 EtOAc/ $\left.\mathrm{CH}_{2} \mathrm{Cl}_{2}\right)$ gave the title compound 9 (69 mg, $0.20 \mathrm{mmol}, 68 \%)$ as a colourless oil. ${ }^{1} \mathrm{H}-\mathrm{NMR}\left(300 \mathrm{MHz}, \mathrm{CDCl}_{3}\right) \delta 7.73(2 \mathrm{H}, \mathrm{d}, J=8.0 \mathrm{~Hz}$, CH), 7.27 (2H, d, $J=8.0 \mathrm{~Hz}, \mathrm{CH}), 4.10-4.00$ (2H, m, CH $\left.\mathbf{H}_{2} \mathrm{OTs}\right), 3.99-3.94$ (1H, m, CHCHOH), $3.80\left(1 \mathrm{H}, \mathrm{t}, J=7.0 \mathrm{~Hz}, \mathrm{CHC}\left(\mathrm{CH}_{3}\right)_{2} \mathrm{OH}\right) 3.66(1 \mathrm{H}, \mathrm{dt}, J=4.0,8.0 \mathrm{~Hz}, \mathrm{CHOH}), 3.26(2 \mathrm{H}, \mathrm{br}$, $\mathrm{OH}), 2.35$ (3H, s, $\left.\mathrm{CH}_{3} \mathrm{Ar}\right), 1.98-1.72$ (4H, m, $\mathrm{CH}_{2}$ (THF)), 1.15 (3H, s, $\left.\left(\mathrm{CH}_{3}\right)_{2} \mathrm{COH}\right) 1.04$ (3H, s, $\left.\left(\mathrm{CH}_{3}\right)_{2} \mathrm{COH}\right) ;{ }^{13} \mathrm{C}-\mathrm{NMR}\left(100 \mathrm{MHz}, \mathrm{CDCl}_{3}\right) \delta 145.3(\mathrm{C}), 133.2(\mathrm{C}), 130.3(\mathrm{CH}), 128.4(\mathrm{CH})$, $86.8(\mathrm{CH}), 78.6(\mathrm{CH}), 72.5(\mathrm{C}), 72.1(\mathrm{CH}), 72.0\left(\mathrm{CH}_{2}\right), 28.6\left(\mathrm{CH}_{2}\right), 28.1\left(\mathrm{CH}_{3}\right), 26.3\left(\mathrm{CH}_{2}\right), 26.1$ $\left(\mathrm{CH}_{3}\right), 22.0\left(\mathrm{CH}_{3}\right)$; IR $v$ 3360(br), 1737(m), 1596(m), 1456(m), 1358(s), 1190(s), 1175(s), 1096(s), 1076(s) cm¹; LRMS (ES+) m/z $711\left(50 \%,[2 \mathrm{M}+\mathrm{Na}]^{+}\right), 367\left(100 \%,[\mathrm{M}+\mathrm{Na}]^{+}\right), 345(25$ $\left.\%,[\mathrm{M}+\mathrm{H}]^{+}\right)$; HRMS (EI) calcd for $\mathrm{C}_{16} \mathrm{H}_{25} \mathrm{O}_{6} \mathrm{~S} \mathrm{~m} / \mathrm{z} 345.1372$, found 345.1359 .

2-(2S*,5R*)-5-[(2R*)Oxiran-2-yl]tetrahydro-2-furanyl-2-propanol (5). To a solution of tosylate 9 (186 mg, $0.54 \mathrm{mmol})$ in $\mathrm{CH}_{2} \mathrm{Cl}_{2}(15 \mathrm{~mL})$ at $0{ }^{\circ} \mathrm{C}$ was added DBU (90 $\left.\mathrm{mg}, 0.59 \mathrm{mmol}\right)$ dropwise by syringe. The reaction was allowed to warm to $\mathrm{rt}$ and stirred for $12 \mathrm{~h}$. Concentration in vacuo gave a yellow oil, which was purified by column chromatography on silica gel (1:40 
$\left.\mathrm{MeOH} / \mathrm{CH}_{2} \mathrm{Cl}_{2}\right)$ to afford the title compound 5 (89 mg, $\left.0.52 \mathrm{mmol}, 96 \%\right)$ as a yellow oil. ${ }^{1} \mathrm{H}-$ NMR $\left(300 \mathrm{MHz}, \mathrm{CDCl}_{3}\right) \delta 4.11(1 \mathrm{H}, \mathrm{ddd}, J=3.0,5.5,7.5 \mathrm{~Hz}, \mathrm{ROCH}), 3.78(1 \mathrm{H}, \mathrm{t}, J=7.0 \mathrm{~Hz}$, $\left.\mathrm{CHC}\left(\mathrm{CH}_{3}\right)_{2} \mathrm{OH}\right), 3.09-3.02\left(2 \mathrm{H}, \mathrm{m}, \mathrm{CHOCH}_{2}, \mathrm{OH}\right), 2.85\left(1 \mathrm{H}, \mathrm{dd}, J=3.0,5.0 \mathrm{~Hz}, \mathrm{CH} \mathbf{H}_{2} \mathrm{OCH}\right)$, $2.79\left(1 \mathrm{H}, \mathrm{dd}, J=4.0,5.0 \mathrm{~Hz}, \mathrm{CH}_{2} \mathrm{OCH}\right), 2.18-1.81\left(4 \mathrm{H}, \mathrm{m}, \mathrm{CH}_{2}(\mathrm{THF})\right), 1.20(3 \mathrm{H}, \mathrm{s}$, $\left.\left(\mathrm{CH}_{3}\right)_{2} \mathrm{COH}\right), 1.05\left(3 \mathrm{H}, \mathrm{s},\left(\mathrm{CH}_{3}\right)_{2} \mathrm{COH}\right) ;{ }^{13} \mathrm{C}-\mathrm{NMR}\left(75 \mathrm{MHz}, \mathrm{CDCl}_{3}\right) \delta 87.1(\mathrm{CH}), 76.7(\mathrm{CH})$, $71.4(\mathrm{C}), 54.7(\mathrm{CH}), 44.3\left(\mathrm{CH}_{2}\right), 29.6\left(\mathrm{CH}_{2}\right), 28.0\left(\mathrm{CH}_{3}\right), 25.8\left(\mathrm{CH}_{2}\right), 25.2\left(\mathrm{CH}_{3}\right)$; IR v 3455(br), 1470(m), 1381(m), 1361(m), 1258(m), 1177(m), 1156(s), 1109(s), 1071(s), 1032(s) $\mathrm{cm}^{-1}$; LRMS (CI) $\mathrm{m} / \mathrm{z} 190\left(25 \%,\left[\mathrm{M}+\mathrm{NH}_{4}\right]^{+}\right), 173\left(20 \%,[\mathrm{M}+\mathrm{H}]^{+}\right), 155\left(100 \%,\left[\mathrm{M}+\mathrm{H}-\mathrm{H}_{2} \mathrm{O}\right]^{+}\right)$; HRMS (CI) calcd for $\mathrm{C}_{9} \mathrm{H}_{17} \mathrm{O}_{3} \mathrm{~m} / \mathrm{z} 173.1178$, found 173.1181 .

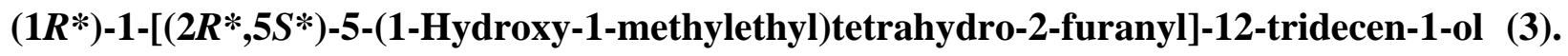
A solution of undec-10-enylmagnesium bromide (11) in THF (3.5 mL of $0.2 \mathrm{M}, 0.7 \mathrm{mmol})$ was added to a suspension of $\mathrm{CuI}(28 \mathrm{mg}, 0.15 \mathrm{mmol})$ in $\mathrm{THF}(10 \mathrm{~mL})$ at $0{ }^{\circ} \mathrm{C}$. After $10 \mathrm{~min}$ stirring, the solution was cooled to $-70{ }^{\circ} \mathrm{C}$ (internal) whereupon it went grey. A solution of epoxide 5 (25 $\mathrm{mg}, 0.14 \mathrm{mmol})$ in THF $(2 \mathrm{~mL})$ was added dropwise. After $45 \mathrm{~min}$ the reaction mixture was quenched by the addition of an aqueous solution of saturated $\mathrm{NH}_{4} \mathrm{Cl} / \mathrm{NH}_{4} \mathrm{OH}(9: 1,30 \mathrm{~mL})$. Ether $(60 \mathrm{~mL})$ was added and the organic phase separated. The organic phase was then washed with brine $(20 \mathrm{~mL})$, dried $\left(\mathrm{Na}_{2} \mathrm{SO}_{4}\right)$ and concentrated in vacuo to give a yellow oil $(250 \mathrm{mg})$. Purification by column chromatography on silica gel (3:7 EtOAc/hexane) gave the title THF diol 3 (32 mg, $0.10 \mathrm{mmol}, 73 \%$ ) as a white solid. Recrystallisation from EtOAc/hexane gave white crystals of mp $30-33{ }^{\circ} \mathrm{C}$; ${ }^{1} \mathrm{H}-\mathrm{NMR}\left(400 \mathrm{MHz}, \mathrm{CDCl}_{3}\right) \delta 5.74(1 \mathrm{H}, \mathrm{tdd}, J=6.5,10.0,17.0 \mathrm{~Hz}$, $\left.\mathrm{CH}=\mathrm{CH}_{2}\right), 4.92(1 \mathrm{H}, \mathrm{dd}, J=2.0,17.0 \mathrm{~Hz}, \mathrm{CHH}=\mathrm{CH}(\mathrm{E})), 4.85(1 \mathrm{H}, \mathrm{dd}, J=2.0,10.0 \mathrm{~Hz}$, $\mathrm{CHH}=\mathrm{CH}(\mathrm{Z})), 3.76(1 \mathrm{H}, \mathrm{q}, J=6.5 \mathrm{~Hz}, \mathrm{CHCHOH}), 3.71\left(1 \mathrm{H}, \mathrm{t}, J=7.0 \mathrm{~Hz}, \mathrm{CHC}\left(\mathrm{CH}_{3}\right)_{2} \mathrm{OH}\right)$, 3.40-3.34 (1H, m, CHOHCH 2$), 2.49$ (2H, br, OH), $1.98\left(2 \mathrm{H}, \mathrm{q}, J=6.5 \mathrm{~Hz}, \mathrm{CH}_{2} \mathrm{CH}=\mathrm{CH}_{2}\right), 1.90-$ 1.61 (4H, m, $\quad \mathrm{CH}_{2} \mathrm{CH}_{2}$ (THF)), $1.20 \quad\left(3 \mathrm{H}, \quad \mathrm{s}, \quad\left(\mathrm{CH}_{3}\right)_{2} \mathrm{COH}\right), \quad 1.48-1.18 \quad(18 \mathrm{H}, \quad \mathrm{m}$, $\left.\left(\mathrm{CH}_{2}\right)_{9} \mathrm{CH}_{2} \mathrm{CH}=\mathrm{CH}_{2}\right), 1.06\left(3 \mathrm{H}, \mathrm{s},\left(\mathrm{CH}_{3}\right)_{2} \mathrm{COH}\right) ;{ }^{13} \mathrm{C}-\mathrm{NMR}\left(100 \mathrm{MHz}, \mathrm{CDCl}_{3}\right) \delta 139.6(\mathrm{CH})$, $114.5\left(\mathrm{CH}_{2}\right), 86.5(\mathrm{CH}), 82.9(\mathrm{CH}), 74.8(\mathrm{CH}), 72.0(\mathrm{C}), 34.6\left(\mathrm{CH}_{2}\right), 34.2\left(\mathrm{CH}_{2}\right), 30.1\left(\mathrm{CH}_{2}\right)$, $30.0\left(\mathrm{CH}_{2}\right), 30.0\left(\mathrm{CH}_{2}\right), 30.0\left(\mathrm{CH}_{2}\right), 29.9\left(\mathrm{CH}_{2}\right), 29.5\left(\mathrm{CH}_{2}\right), 29.3\left(\mathrm{CH}_{2}\right), 28.8\left(\mathrm{CH}_{2}\right), 27.9$ $\left(\mathrm{CH}_{3}\right), 26.5\left(\mathrm{CH}_{2}\right), 26.1\left(\mathrm{CH}_{2}\right), 25.5\left(\mathrm{CH}_{3}\right)$; IR $v$ 3376(br), 1640(m), 1463(s), 1377(m), 1362(m), 1162(s), 1081(s), 995(s) $\mathrm{cm}^{-1}$; LRMS (ES+) m/z 675 (50\%, [2M+Na] $\left.]^{+}\right), 653\left(5 \%,[2 \mathrm{M}+\mathrm{H}]^{+}\right)$, $349\left(100,[\mathrm{M}+\mathrm{Na}]^{+}\right)$; HRMS (EI) calcd for $\mathrm{C}_{20} \mathrm{H}_{39} \mathrm{O}_{3} \mathrm{~m} / \mathrm{z}$ 327.2899, found 327.2901.

(5S)-3-(E,13R*)-13-Hydroxy-13-[(2R*,5S*)-5-(1-hydroxy-1-methylethyl)tetrahydro-2-

furanyl]-2-tridecenyl-5-methyl-2,5-dihydro-2-furanone (10). Under an atmosphere of argon, a bright orange solution of the ruthenium complex 12 (3 $\mathrm{mg}, 0.02 \mathrm{mmol}, 5 \mathrm{~mol} \%$ ) in degassed $\mathrm{CH}_{3} \mathrm{OH}(1 \mathrm{~mL})$ was added by syringe to a stirred solution of alkene 3 (56 mg, $\left.0.17 \mathrm{mmol}\right)$ and alkyne $4(24.5 \mathrm{mg}, 0.17 \mathrm{mmol})^{19}$ in degassed $\mathrm{CH}_{3} \mathrm{OH}(4 \mathrm{~mL})$. The solution was heated at reflux for $2.5 \mathrm{~h}$. After cooling to $\mathrm{rt}$ the mixture was diluted with ether $(30 \mathrm{~mL})$, filtered and concentrated in vacuo to give an orange oil $(75 \mathrm{mg})$. Purification by column chromatography on silica gel (1:40 $\left.\mathrm{CH}_{3} \mathrm{OH} / \mathrm{CH}_{2} \mathrm{Cl}_{2}\right)$, gave butenolide 10 (30 $\left.\mathrm{mg}, 0.07 \mathrm{mmol}, 42 \%\right)$ as a yellow oil. ${ }^{1} \mathrm{H}-\mathrm{NMR}\left(400 \mathrm{MHz}, \mathrm{CDCl}_{3}\right) \delta 6.99\left(1 \mathrm{H}, \mathrm{d}, J=1.5 \mathrm{~Hz}, \mathrm{CH}=\mathrm{CCO}_{2}\right), 5.56(1 \mathrm{H}, \mathrm{dt}, J=15.1,6.5$ $\left.\mathrm{Hz}, \mathrm{CH}=\mathrm{CHCH}_{2} \mathrm{CCO}_{2}\right), 5.46\left(1 \mathrm{H}, \mathrm{dt}, J=15.1,6.5 \mathrm{~Hz}, \mathrm{CHCH}_{2} \mathrm{CCO}_{2}\right), 5.01(1 \mathrm{H}, \mathrm{dq}, J=1.5,6.5$ 
$\left.\mathrm{Hz}, \mathrm{CHCH}_{3}\right), 3.83(1 \mathrm{H}, \mathrm{q}, J=6.2 \mathrm{~Hz}, \mathrm{CHCHOH}), 3.76\left(1 \mathrm{H}, \mathrm{t}, J=7.0 \mathrm{~Hz}, \mathrm{CHC}\left(\mathrm{CH}_{3}\right)_{2} \mathrm{OH}\right), 3.44$ $(1 \mathrm{H}, \mathrm{q}, J=5.5 \mathrm{~Hz}, \mathrm{CHOH}), 2.94\left(2 \mathrm{H}, \mathrm{d}, J=6.5 \mathrm{~Hz}, \mathrm{CH}_{2} \mathrm{CCO}_{2}\right), 2.50$ (2H, br, OH), 2.04 (2H, q, $\left.J=6.6 \mathrm{~Hz}, \mathrm{CH}_{2} \mathrm{CH}=\mathrm{CHCCO}_{2}\right), 1.96-1.69\left(4 \mathrm{H}, \mathrm{m}, \mathrm{CH}_{2} \mathrm{CH}_{2}(\mathrm{THF})\right), 1.40(3 \mathrm{H}, \mathrm{d}, J=6.5 \mathrm{~Hz}$, $\left.\mathrm{CH}_{3} \mathrm{CH}\right), 1.27\left(3 \mathrm{H}, \quad \mathrm{s},\left(\mathrm{CH}_{3}\right)_{2} \mathrm{COH}\right), 1.51-1.25\left(16 \mathrm{H}, \mathrm{m},\left(\mathbf{C H}_{2}\right)_{8} \mathrm{CHOH}\right), 1.13(3 \mathrm{H}, \mathrm{s}$, $\left.\left(\mathrm{CH}_{3}\right)_{2} \mathrm{COH}\right) ;{ }^{13} \mathrm{C}-\mathrm{NMR}\left(100 \mathrm{MHz}, \mathrm{CDCl}_{3}\right) \delta 173.6(\mathrm{C}), 149.5(\mathrm{CH}), 134.3(\mathrm{CH}), 133.7(\mathrm{C})$, $124.4(\mathrm{CH}), 86.3(\mathrm{CH}), 82.6(\mathrm{CH}), 77.7(\mathrm{CH}), 74.6(\mathrm{CH}), 71.7(\mathrm{C}), 34.3\left(\mathrm{CH}_{2}\right), 32.6\left(\mathrm{CH}_{2}\right), 29.8$ $\left(\mathrm{CH}_{2}\right), 29.7\left(\mathrm{CH}_{2}\right), 29.7\left(\mathrm{CH}_{2}\right), 29.5\left(\mathrm{CH}_{2}\right), 29.4\left(\mathrm{CH}_{2}\right), 29.3\left(\mathrm{CH}_{2}\right), 28.5\left(\mathrm{CH}_{2}\right), 28.5\left(\mathrm{CH}_{2}\right), 27.6$ $\left(\mathrm{CH}_{3}\right), 26.2\left(\mathrm{CH}_{2}\right), 25.9\left(\mathrm{CH}_{2}\right), 25.2\left(\mathrm{CH}_{3}\right), 19.3\left(\mathrm{CH}_{3}\right)$; IR $v$ 3420(br), 2849(s), 1739(s), 1462(s), 1376(m), 1240(s), 1084(m) $\mathrm{cm}^{-1}$; LRMS (ES+) m/z $868\left(30 \%,[2 \mathrm{M}+\mathrm{Na}]^{+}\right), 445(100 \%$, $\left.[\mathrm{M}+\mathrm{Na}]^{+}\right), 423\left(25 \%,[\mathrm{M}+\mathrm{H}]^{+}\right)$; HRMS (ES+) calcd for $\mathrm{C}_{25} \mathrm{H}_{42} \mathrm{O}_{5} \mathrm{Na} \mathrm{m} / \mathrm{z}$ 445.2924, found 445.2923.

Compounds 2 \& 13. In a steel bomb were placed butenolide 10 (30 mg, $0.07 \mathrm{mmol})$ and Wilkinson's catalyst ( $7 \mathrm{mg}, 0.007 \mathrm{mmol})$ in a 1:1 solution of benzene/ethanol $(3 \mathrm{~mL})$. After 3 cycles of nitrogen/evacuation, $\mathrm{H}_{2}$ (2-4 bar) was introduced. The mixture was stirred at $\mathrm{rt}$ for $18 \mathrm{~h}$. After releasing the pressure, $\mathrm{CHCl}_{3}(10 \mathrm{~mL})$ was added and the mixture filtered, resulting in a brown liquor that was concentrated in vacuo and then purified by column chromatography on silica gel (50\% EtOAc/hexane). This gave a yellow oil that contained butenolide 2 and the over reduced lactone $\mathbf{1 3}$ as a 3:2 inseparable mixture (28 $\mathrm{mg}, 95 \%)$.

Compound 2 (selected data): ${ }^{1} \mathrm{H}-\mathrm{NMR}\left(300 \mathrm{MHz}, \mathrm{CDCl}_{3}\right) \delta 6.99(1 \mathrm{H}, \mathrm{d}, J=1.5 \mathrm{~Hz}$, $\left.\mathrm{CH}=\mathrm{CCO}_{2}\right), 4.99\left(1 \mathrm{H}, \mathrm{dq}, J=1.5,6.5 \mathrm{~Hz}, \mathrm{CHCH}_{3}\right), 3.84(1 \mathrm{H}, \mathrm{q}, J=6.2 \mathrm{~Hz}, \mathrm{CHCHOH}), 3.76$ $\left(1 \mathrm{H}, \mathrm{t}, J=7.0 \mathrm{~Hz}, \mathrm{CHC}\left(\mathrm{CH}_{3}\right)_{2} \mathrm{OH}\right), 3.44(1 \mathrm{H}, \mathrm{q}, J=5.5 \mathrm{~Hz}, \mathrm{CHOH}), 2.24(2 \mathrm{H}, \mathrm{t}, J=7.4 \mathrm{~Hz}$, $\left.\mathrm{CH}_{2} \mathrm{CCO}_{2}\right) ;{ }^{13} \mathrm{C}-\mathrm{NMR}\left(75 \mathrm{MHz}, \mathrm{CDCl}_{3}\right) \delta 173.9(\mathrm{C}), 148.9(\mathrm{CH}), 134.3(\mathrm{CH}), 86.2(\mathrm{CH}), 82.6$ $(\mathrm{CH}), 75.1(\mathrm{CH}), 74.4(\mathrm{CH})$; LRMS (ES+) of mixture m/z $447\left(100 \%,[\mathrm{M}+\mathrm{Na}]^{+}\right), 425(65 \%$, $\left.[\mathrm{M}+\mathrm{H}]^{+}\right)$.

Compound 13 (selected data): ${ }^{1} \mathrm{H}-\mathrm{NMR}\left(300 \mathrm{MHz}, \mathrm{CDCl}_{3}\right) \delta$ 4.51-4.41 $\left(1 \mathrm{H}, \mathrm{m}, \mathrm{CHCH}_{3}\right)$, $3.84(1 \mathrm{H}, \mathrm{q}, J=6.2 \mathrm{~Hz}, \mathrm{CHCHOH}), 3.76\left(1 \mathrm{H}, \mathrm{t}, J=7.0 \mathrm{~Hz}, \mathrm{CHC}\left(\mathrm{CH}_{3}\right)_{2} \mathrm{OH}\right), 3.44(1 \mathrm{H}, \mathrm{q}, J=$ $5.5 \mathrm{~Hz}, \mathrm{CHOH})$ (THF protons coincidental for both compounds); LRMS (ES+) of mixture $\mathrm{m} / \mathrm{z}$ $449\left(70 \%,[\mathrm{M}+\mathrm{Na}]^{+}\right), 427\left(40 \%,[\mathrm{M}+\mathrm{H}]^{+}\right)$.

\section{Acknowledgements}

We thank the Royal Society for a university research fellowship (RCDB) and Merck Sharp \& Dohme and Pfizer for unrestricted grants.

\section{References and Notes}

F Ethyl (4S)-4-hydroxy-2-pentynoate (4) was prepared by the method of Trost et al. ${ }^{19}$ Spectroscopic data agreed with that published. ${ }^{19}$ Optical purity was assessed by polarimetry 
$[\alpha]^{20}{ }_{\mathrm{D}}-26.7^{\circ}\left(\right.$ c. $\left.0.232, \mathrm{CHCl}_{3}\right)$ Lit. $[\alpha]^{25}{ }_{\mathrm{D}}-28.4^{\circ}$ (c. $\left.0.206, \mathrm{CHCl}_{3}\right) .^{19}$

${ }^{\dagger}$ Only one diastereoisomer for each compound (2 and 13) is shown in scheme 3.

1. Alali, F. Q.; Liu, X. X.; McLaughlin, J. L. J. Nat. Prod. 1999, 62, 504.

2. Oberlies, N. H.; Jones, J. L.; Corbett, T. H.; Fotopoulos, S. S.; McLaughlin, J. L. Cancer Lett. 1995, 96, 55.

3. Morre, D. J.; Decabo, R.; Farley, C.; Oberlies, N. H.; McLaughlin, J. L. Life Sci. 1994, 56, 343.

4. Zeng, L.; Ye, Q.; Oberlies, N. H.; Shi, G.; Gu, Z. M.; He, K.; McLaughlin, J. L. Nat. Prod. Rep. 1996, 13, 275.

5. Fang, X. P.; Rieser, M. J.; Gu, Z. M.; Zhao, G. X.; McLaughlin, J. L. Phytochem. Anal. 1993, 4, 27.

6. Rupprecht, J. K.; Hui, Y. H.; McLaughlin, J. L. J. Nat. Prod. 1990, 53, 237.

7. For lead references to Annonaceous acetogenin syntheses prior to 1998 see ref. 1. For selected strategies recently used in total syntheses of Annonaceous acetogenins see: (a) Albarella, L.; Musumeci, D.; Sica, D. Eur. J. Org. Chem. 2001, 997. (b) Avedissian, H.; Sinha, S. C.; Yazbak, A.; Sinha, A.; Neogi, P.; Keinan, E. J. Org. Chem. 2000, 65, 6035. (c) Dixon, D. J.; Ley, S. V.; Reynolds, D. J. Angew. Chem.-Int. Ed. 2000, 39, 3622. (d) Hoppen, S.; Baurle, S.; Koert, U. Chem.-Eur. J. 2000, 6, 2382. (e) Hu, T. S.; Yu, Q.; Wu, Y. L.; Wu, Y. K. J. Org. Chem. 2001, 66, 853. (f) Kuriyama, W.; Ishigami, K.; Kitahara, T. Heterocycles 1999, 50, 981. (g) Makabe, H.; Tanaka, A.; Oritani, T. Tetrahedron 1998, 54, 6329. (h) Marshall, J. A.; Jiang, H. J. J. Org.Chem. 1999, 64, 971. (i) Neogi, P.; Doundoulakis, T.; Yazbak, A.; Sinha, S. C.; Keinan, E. J. Am. Chem. Soc. 1998, 120, 11279. (j) Rassu, G.; Zanardi, F.; Battistini, L.; Casiraghi, G. Chem. Soc. Rev. 2000, 29, 109. (k) Takahashi, S.; Maeda, K.; Hirota, S.; Nakata, T. Org. Lett. 1999, 1, 2025. (1) Wang, Z. M.; Tian, S. K.; Shi, M. Eur. J. Org. Chem. 2000, 349. (m) Yu, Q.; Wu, Y. K.; Ding, H.; Wu, Y. L. J. Chem. Soc.-Perkin Trans. 1 1999, 1183.

8. Hoppen, S.; Emde, U.; Friedrich, T.; Grubert, L.; Koert, U. Angew. Chem.Int. Ed. 2000, 39, 2099.

9. Zeng, B. B.; Wu, Y. K.; Yu, Q.; Wu, Y. L.; Li, Y.; Chen, X. G. Angew. Chem.-Int. Ed. 2000, 39, 1934.

10. Kuwabara, K.; Takada, M.; Iwata, J.; Tatsumoto, K.; Sakamoto, K.; Iwamura, H.; Miyoshi, H. Eur. J. Biochem. 2000, 267, 2538.

11. Baurle, S.; Peters, U.; Friedrich, T.; Koert, U. Eur. J. Org. Chem. 2000, 2207.

12. D'Souza, L. J.; Sinha, S. C.; Lu, S. F.; Keinan, E. Tetrahedron 2001, 57, 5255.

13. Baldwin, J. E.; Crossley, M. J.; Lehtonen, E.-M. Chem. Commun. 1979, 918.

14. Klein, E.; Rojahn, W. Tetrahedron 1965, 21, 2353.

15. Walba, D. M.; Wand, M. D.; Wilkes, M. C. J. Am. Chem. Soc. 1979, 101, 4396.

16. The permanganate oxidative cyclisation of 1,5-dienes has previously been employed to aid the stereochemical assignment of acetogenins: (a) Gale, J. B.; Yu, J. G.; Hu, X. F. E.; Khare, A.; Ho, D. K.; Cassady, J. M. Tetrahedron Lett. 1993, 34, 5847. (b) Bertrand, P.; Gesson, J. 
P. Tetrahedron Lett. 1992, 33, 5177.

17. Walba, D. M.; Przybyla, C. A.; Walker, C. B. J. Am. Chem. Soc. 1990, 112, 5624.

18. Kocienski, P. J.; Brown, R. C. D.; Pommier, A.; Procter, M.; Schmidt, B. J. Chem. Soc.Perkin Trans. 1 1998, 9.

19. Trost, B. M.; Muller, J. J.; Martinez, J. J. Am. Chem. Soc. 1995, 117, 1888.

20. Marbet, R.; Saucy, G. Helv. Chim. Acta. 1967, 50, 2091.

21. Stephan, E.; Pourcelot, G.; Cresson, P. Chem. Ind. 1988, 562.

22. For an application of the permanganate oxidative cyclisation towards the synthesis of acetogenin fragments see: Brown, R. C. D.; Hughes, R. M.; Keily, J.; Kenney, A. Chem. Commun. 2000, 1735.

23. Related reductions of disubstituted alkenes and alkynes have been reported during previous total syntheses of acetogenins. Hoye, T. R.; Ye, Z. X. J. Am. Chem. Soc. 1996, 118, 1801.

24. Perrin, D. D.; Armarego, W. L. F. Purification of laboratory chemicals, 3rd Edn; Butterworth-Heinemann Ltd.: Oxford, 1994. 